Obituary

\title{
In memory of Toshihico Arimitsu
}

Professor Toshihico Arimitsu, a world renowned expert in the theory of non-equilibrium statistical physics, died on 30 September 2016 in his home in Japan after a long struggle with a heart disease.

Toshihico Arimitsu was born on 8 February 1952 in Tokyo. He graduated from the University of Tokyo, BSc in 1975 and MSc in 1977, and received his PhD in 1980 under the supervision of Professor Masuo Suzuki. He was appointed Research Associate of Physics in the Faculty of Pure and Applied Science at the University of Tsukuba the same year. After promotions to Lecturer in 1982 and to Associate Professor in 1994 he became Professor in 1999. For relatively short periods of time he was also a visiting researcher at the University of Alberta in 1984-1985 and a visiting professor in 1986. As a recognition of his expertise, he was invited to join the Editorial Board of Condensed Matter Physics in 1995. He remained on the journal as Editor, a position he held at the time of his death, through which he maintained an active interest in the continued success of the periodical for more than twenty years. Because of the illness, Professor Toshihico Arimitsu retired from the University of Tsukuba in 2015.

Toshihico Arimitsu's studies during his doctoral period [1], in collaboration with Fumiaki Shibata, Professor at the Ochanomizu University, Japan, developed into a damping theoretical formulation for non-equilibrium quantum systems. The method was applied to obtain rigorous solutions of the optical parametric amplification, exchange dephasing and laser oscillation. This work has been highly appreciated in the field of non-equilibrium quantum statistical physics. It also greatly influenced the Toshihico Arimitsu's career in a sense that fundamental aspects of non-equilibrium quantum statistical physics became the main subject of his research work for many years. One example is given by his contribution to the comprehension of nonlinear transient optical processes [2], in collaboration with Masashi Ban, Professor at the Ochanomizu University, Japan.

In 1984 Toshihico Arimitsu started developing a general framework for non-equilibrium quantum systems, called non-equilibrium thermo field dynamics (NETFD) [3--5], in collaboration with Hiroomi Umezawa, Professor at the University of Alberta, Canada. NETFD is a canonical operator formalism which provides a method to treat dissipative quantum systems in a manner that is similar to the usual quantum field theory and is a generalization of thermo field dynamics by Takahashi and Umezawa to non-equilibrium systems [6]. It presents a new description of dissipative processes and uses two kinds of operators, non-tilde and tilde ones, condensing into an unstable vacuum. The formalism has been used, in particular, to study time-dependent behavior of non-equilibrium quantum systems involving stochastic forces which can be boson or fermion type [7], or to model a noisy-channel which is under the influence of spatially correlated quantum Brownian motion [8]. In the former case, it leads to a unified system of quantum stochastic differential equations under the action of quantum Brownian motion, including the quantum stochastic Liouville equation and the quantum Langevin equation. In addition, the quantum Fokker-Planck equation is derived by taking the random average of the corresponding stochastic Liouville equation. In the latter case, it shows that errors due to spatially correlated noises can be corrected by the quantum error-correction code and error-correction procedure prepared for those for independent noises.

Toshihico Arimitsu's contributions were not restricted to quantum systems only. Some examples of classical systems include, in particular, studies on a universality of period doubling bifurcations in low dimensional chaotic dynamical systems [9], in collaboration with Takumi Motoike, Professor at the Surugadai University, Japan, and multifractal probability density function analysis on intermittency of turbulence [10], in collaboration with his wife Naoko Arimitsu, Professor at the Yokohama National University, Japan. The former considers a non-autonomous system and introduces a set of new concepts named irreducible and reducible templates. The notion of the irreducible template is important in the unique representation of a period doubling sequence. The latter, to explain the probability density function of velocity fluctuation observed in experiments and numerical simulations of turbulence, employs the 
distribution function based on non-extensive Tsallis statistics. Regarding turbulence, Toshihico Arimitsu also contributed to statistical laws of quantum fluid turbulence [11] in collaboration with Kyo Yoshida, Assistant Professor at the University of Tsukuba, Japan.

Courageous as a scientist, Toshihico Arimitsu was willing to move into entirely new areas, to learn and teach new ideas, and even to learn a little the information theory. In 2000 he started to collaborate with Petr Jizba, then a foreign professor at Tsukuba University, on topics lying at the interface between statistical physics and information theory. In the years 2001-2005, together they developed a full-fledged statistical physics of multifractal systems based on Rényi's information measure. They also substantially promoted the concept of Rényi entropy as a viable alternative to non-extensive entropy of Tsallis [12]. This seminal paper is now, with more than 100 citations, a classic in the field. In the last collaborative research study, Arimitsu and Jizba introduced a new hybrid entropy that interpolates in its axiomatics between extensive entropy of Rényi and non-extensive Tsallis entropy. In recent years, this so-called Arimitsu-Jizba hybrid entropy started to live a life of its own by demonstrating its relevance in statistical systems with large spatial correlations and non-trivial configuration spaces.

Toshihico Arimitsu was always enthusiastic about fundamental issues and novel ideas. He was actively promoting his own concepts and diligently encouraging young researchers to develop their own visions. Such a policy has found a reflection in the series of symposiums on non-equilibrium statistical physics organized by Arimitsu every year from 1992 till 2005. The symposiums were not restricted to specific topics or projects but were open to problems from various fields, so that the participants with different specialties could exchange and disseminate their ideas.

With Toshihico Arimitsu early passing, the community of statistical physics has lost a unique theoretical physicist. However, his influence lives on. In his quiet, polite, attentive, and kind way, Toshihico Arimitsu won the respect and appreciation of his colleagues and friends. He will be remembered as an accomplished scientist, a devoted mentor, a gifted author, and a good friend.

M. Ban (Graduate School of Humanities and Sciences, Ochanomizu University, Tokyo, Japan)

T. Hayashi (R\&D Department, Engineering \& Operation Divison, MCC Corporation, Tokyo, Japan)

P. Jizba (Department of Physics, Czech Technical University in Prague, Prague, Czech Republic)

A.E. Kobryn (National Institute for Nanotechnology, Edmonton, Canada)

T. Motoike (Faculty of Media and Information Resources, Surugadai University, Hanno, Japan)

F. Shibata (Graduate School of Humanities and Sciences, Ochanomizu University, Tokyo, Japan)

K. Yoshida (Faculty of Pure and Applied Science, University of Tsukuba, Tsukuba, Japan)

\section{References}

1. Shibata F., Arimitsu T., J. Phys. Soc. Jpn., 1980, 49, No. 3, 891-897, doi 10.1143/JPSJ.49.891

2. Ban M., Arimitsu T., J. Phys. Soc. Jpn., 1986, 55, No. 5, 1759-1777, doi $10.1143 /$ JPSJ.55.1759

3. Arimitsu T., Umezawa H., Prog. Theor. Phys., 1985, 74, No. 2, 429-432, doi 10.1143/PTP.74.429

4. Arimitsu T., Umezawa H., Prog. Theor. Phys., 1987, 77, No. 1, 32-52, doi 10.1143/PTP.77.32

5. Arimitsu T., Umezawa H., Prog. Theor. Phys., 1987, 77, No. 1, 53-67, doi 10.1143/PTP.77.53

6. Arimitsu T., Condens. Matter Phys., 1994, 4, 26-88, doi:10.5488/CMP.4.26

7. Kobryn A.E., Hayashi T., Arimitsu T., Ann. Phys., 2003, 308, No. 2, 395-446, doi $10.1016 /$ S0003-4916(03)00178-7.

8. Arimitsu T., Interdiscip. Inf. Sci., 2009, 15, No. 3, 441-471, doi 10.4036/iis.2009.441.

9. Arimitsu T., Motoike T., Physica D, 1995, 84, No. 1-2, 290-302, doi:10.1016/0167-2789(95)00032-Y

10. Arimitsu N., Arimitsu T., Physica A, 2011, 390, No. 2, 161-176, doi 10.1016/j.physa.2010.09.036

11. Yoshida K., Arimitsu T., J. Phys. A: Math. Theor., 2013, 46, No. 33, 335501, doi $10.1088 / 1751-8113 / 46 / 33 / 335501$

12. Jizba P., Arimitsu T., Ann. Phys., 2004, 312, No. 1, 17-59, doi $10.1016 /$ j.aop.2004.01.002 